\title{
Targeting rictor inhibits mouse vascular tumor cell proliferation and invasion in vitro and tumor growth in vivo
}

\author{
N. N. ZHENG ${ }^{1}$, X. D. DING ${ }^{2}$, H. P. ZHANG ${ }^{1, *}$ \\ ${ }^{1}$ Department of Pathophysiology, China Medical University, Shenyang, China, 110001; ${ }^{2}$ Department of anesthesiology, Shengjing Hospital \\ of China Medical University, Shenyang, China, 110004
}

*Correspondence: hpzhangsq@yahoo.com.cn

Received May 16, 2012 / Accepted June 20, 2012

\begin{abstract}
Vascular tumor is an abnormal buildup of blood vessels in the skin or internal organs that can lead to disfigurement and/or life-threatening consequences. The mechanism of hemangiogenesis remains unknown. The aim of this study was to assess the role of rapamycin-insensitive companion of mTOR (Rictor) in control of vascular tumor malignant biological behavior and cell signaling mechanism in Mouse Hemangioendothelioma Endothelial Cells (EOMA cells) and nude mouse model. Knocking down rictor was mediated by lentivirus shRNA. The role and mechanism of rictor in vascular tumor were assessed by western blotting, wst-1 proliferation assay, matrigel invasion assay and xenograft vascular tumor growth. Our results in vitro showed that loss of rictor down-regulated phosphorylation of AKT and S6 by which EOMA cells growth and proliferation were greatly suppressed. Knock down of rictor also inhibited the invasion of EOMA cells. Furthermore, we demonstrated that knock down of rictor inhibited xenograft vascular tumor growth in nude mice. Taken together, we purpose that rictor contributed to vascular tumor growth and progression. Targeting rictor becomes an effective strategy in vascular tumor treatment.
\end{abstract}

Key words: AKT, mTOR2, rictor, vascular tumor, hemangioma

Vascular tumors are abnormal angiogenic proliferations of neoplastic endothelial cells (EC) with a wide spectrum of clinical diseases ranging from common hemangioma in children to malignant angiosarcoma in adults. Vascular tumors and related vascular malformations have been found in rare genetic syndromes such as PHACES, Kasabach-Merritt, Sturge-Weber and Klippel-Trenaunay syndromes, which have developmental defects in multiple tissues and organs [1-3]. To date, the cellular and molecular mechanisms leading to vascular tumor formation are poorly understood and underinvestigated. Pathological angiogenesis is a major biological process in vascular tumor formation[1].

The phophatidylinositol-3-kinase (PI3k)/AKT signaling pathway is a major pathway activated by vascular endothelial growth factor (VEGF) that regulates EC survival and angiogenesis, and appears to be altered in endothelial cell tumors[4,5]. In infantile hemangioma, tumor EC have increased proliferation and migration in response to VEGF as compared to normal EC[6,7]. Human angiosarcoma expresses VEGF-A, VEGFR-1, VEGFR-2 and VEGFR-3, and hyperac- tivation of the PI3K pathway results in hemangiosarcoma formation in chicken chorioallantoic membrane[8-11]. Similarly, Kaposi's sarcoma, a vascular tumor commonly occurs in HIV/AIDS, has been shown to express high levels of VEGF, and PI3k/AKT activation in endothelial cells infected by Kaposi's sarcoma-associated herpesvirus confers a survival advantage $[12,13]$.

The mammalian target of rapamycin (mTOR), a serinethreonine kinase, acts as a central regulator of the PI3K/AKT signaling pathway[14]. It has been involved in many pathophysiological processes and especially in cancer[15]. Blocking mTOR creates a starvation-like effect in vascular tumor cells by interfering with endothelial cell growth, division, metabolism and angiogenesis.

mTOR exists in two distinct complexes, raptor-mTOR (mTORC1) and rictor-mTOR(mTORC2), which have different functions. Raptor-mTOR, which is composed of mTOR, G $\beta \mathrm{L}$, PRAS40, deptor and raptor, locates at downstream of AKT and regulates cell growth and proliferation. Rictor-mTOR, which contains mTOR, G $\beta$ L, mSIN1, deptor, protor-1 and 
rictor, is a upstream complex and regulates cell survival and cytoskeletal organization through the phosphorylation AKT at Thr473 and PKCa respectively. The role of mTORC1 in cancer progression has been extensively characterized, whereas the role of mTORC2 is much less documented[16]. Rictor originally identified as a regulator of cytoskeletal dynamics, is also an important component of mTORC2. The activation of the mTOR2 complex depends on the presence of the rictor protein. It is necessary for Ser 473 phosphorylation and that a reduction in rictor expression inhibited an AKT effector. Depletion of rictor in breast and prostate cancer cell lines resulted in inhibition of AKT Ser(473) phosphorylation and induction of apoptosis.

So far it has been shown that targeting rictor might be beneficial in tumors harboring high levels of activated AKT, such as gliomas and vascular tumors. We wish here to analyze the role of rictor in hemangioma.

\section{Material and methods}

Cell culture. HEK 293T cells and EOMA cells were cultured at $37^{\circ} \mathrm{C}$ and $5 \% \mathrm{CO}_{2}$ in Dulbecco's modified Eagle's medium (DMEM) with $4.5 \mathrm{~g} /$ /iter glucose supplemented with $10 \%$ fetal bovine serum (FBS) and antibiotics. Primary mouse heart endothelial cells and lung endothelial cells were kindly separated and provided by Carole Perruzzi. EOMA cells about $80 \%$ confluence were washed with PBS and serum-starved with DMEM $/ 0.1 \%$ FBS for 18 hours. Rapamycin(10nM) was treaded for overnight.

Lentivirus-mediated short hairpin RNA silencing. Mouse targeting rictor short-hairpin RNA (shRNA) oligonucleotides were cloned into the PLKO lentiviral expression system which were provided from LeslieWardwell, RNAi Facility, Dana-Farber Cancer Institute. The oligonucleotides encoding the shRNAs expression cassettes targeting mouse rictor were as follows: (shrictor: GCGGTTCATACAAGAGTTATT). To generate lentivirus particles, a $400-\mu l$ transfection mixture containing shrictor, PAX2, VSVG and a transfecting reagent polyethylenimine (Polysciences) in DMEM media was incubated for 15 minutes at room temperature, and then added to HEK $293 \mathrm{~T}$ cells $\left(3 \times 10^{6}\right.$ cells per $6-\mathrm{cm}$ plate) in suspension in $3 \mathrm{ml} \mathrm{DMEM} / 10 \%$ FBS. Cells were incubated at $37^{\circ} \mathrm{C}$ in viral incubator for 48 hours. Media supernatant containing lentivirus particles was collected and filtered through a $0.45-\mu \mathrm{m}$ syringe filter. For viral transduction, hexadimethrine bromide (polybrene, $5 \mu \mathrm{g} / \mathrm{ml}$ ) (Sigma) was added to viral supernatant, which was then transferred directly to EOMA cells $\left(1.5 \times 10^{6}\right.$ cells per $6-\mathrm{cm}$ plate). Four hours later, additional DMEM/10\%FBS was added to the cells to dilute polybrene and cells were cultured overnight. Transduced cells were subsequently selected in puromycin ( $2 \mathrm{ug} / \mathrm{ml})$ for 48 hours.

Immunoblotting. Cells were washed in ice-cold phosphatebuffered saline and whole-cell lysates were homogenized in lysis buffer (1ml RIPA buffer [Tris-HCL(50Mm,PH,7.4),NACL(1
50mM),NP-40(1\%),Sodium deoxycholate(0.5\%),SDS(0.1\%)], $10 \mu \mathrm{L}$ Protease

Inhibitor Cocktail [Sigma], and $10 \mu \mathrm{L}$ Phosphatase Inhibitor

Cocktail I and II [Sigma]). Cell lysates were sonicated two times for $15 \mathrm{~s}$ and centrifuged at $14,000 \mathrm{~g}$ for $15 \mathrm{~min}$ at $4^{\circ} \mathrm{C}$. Cleared lysates were then used to determine total protein levels (Broadford Protein Assay, Eppendorf). After dilution with sample buffer (Laemmli's SDS-Sample Buffer, Boston BioProducts), equal protein amounts (typically 20ug) were loaded onto $4-20 \%$ Tris-Glycine gels. Proteins were transferred to nitrocellulose membranes (BIO-RAD). Nonspecific reactions were blocked for $1 \mathrm{~h}$ in 5\% dry milk solution in Tris-buffered saline plus $0.1 \%$ Tween-20(TBST) and then incubated with the appropriate primary antibody at an appropriate dilution overnight at $4{ }^{\circ} \mathrm{C}$ in $5 \%$ BSA in TBST. Membranes were washed three times in TBST followed by incubation with the appropriate secondary horseradish peroxidase (HRP)-linked antibodies and again washed three times. Membranes were developed with enhanced chemiluminescence reagents (Thermo Scientific, Rockford, IL) and exposed to film.

Animals and tumor growth. Four- to six-week-old female $\mathrm{Nu} / \mathrm{Nu}$ mice (Charles River, $\mathrm{MA}$ ) were used in our experiments. EOMA PLKO, shrictor cells $\left(2 \times 10^{5}\right.$ cells per site) were injected subcutaneously in the flank of mice (2 sites/mouse, 5 mice/group). Tumor growth was measured daily with a caliper. The greatest longitudinal diameter (length) and the greatest transverse diameter (width) were determined. Tumor volume based on caliper measurements were calculated by the formula: tumor volume $=1 / 2\left(\right.$ length $\times$ width $\left.^{2}\right)$. Day 13 after injection tumors were taken for histologic and immunoblotting studies. All studies were conducted in compliance with the Beth Israel Deaconess Medical Center IACUC guidelines.

Antibodies and reagents: all antibodies were purchased from Cell Signaling Technology. Rapamycin was from Alexis Corporation (San Diego, Calif).

Proliferation assay. Cell proliferation assay was done according to the directions of the manufacturer (Roche Applied Science). Knocking down rictor EOMA cells and PLKO control EOMA cells 100ul each well in 96-well plates were cultured for 48h. $10 \mu \mathrm{L}$ of WST-1 reagent was added to each well and cells were incubated for $4 \mathrm{~h}$, after which the absorbance of the samples was measured against a background control as blank at $450 \mathrm{~nm}$ using a microplate reader(Molecular Device, California).

Cell invasion assay. Cell invasion assays were performed in duplicates. $100 \mu \mathrm{l}$ of Growth Factor-Reduced Matrigel (BD Biosciences) was added into upper chamber of transwell and incubated for 4 hours for gelling at $37^{\circ} \mathrm{C}$ and $5 \% \mathrm{CO}_{2}$. 100ul of Cells $\left(1 \times 10^{5}\right)$ resuspended in DMED/0.1\% FBS were added onto the matrigel. Lower chamber was filled with $600 \mathrm{ul}$ of DMEM $/ 10 \% \mathrm{FBS}$, then cells were incubated at $37^{\circ} \mathrm{C}$ for 20-24 hours followed by cell staining with Diff-Quick and counted. The invasion index was calculated by the ratio of invasive EOMA cells with rictor scilencing and PLKO control. 


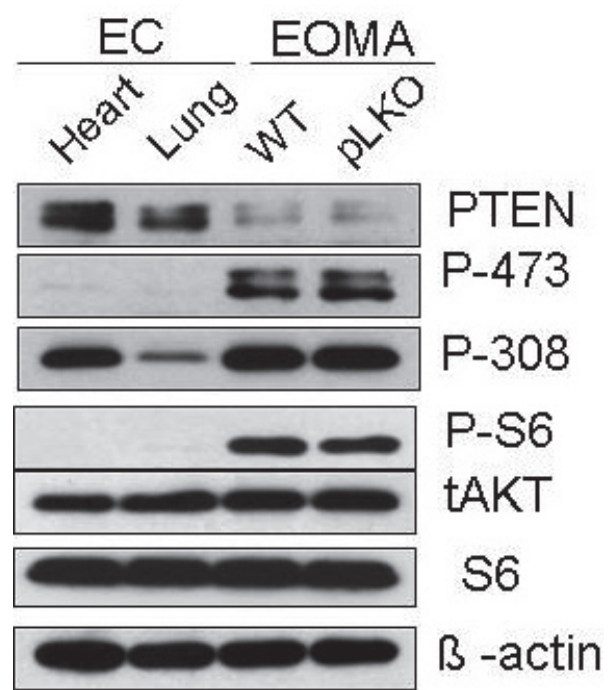

Figure 1. Western blot analysis of normal mouse ECs and EOMA cells for PI3K/AKT signaling pathway. Increased phospho-S6, phosphoralated AKT and decreased PTEN occured with B-actin as protein loading control.

Statistical Analysis. Results are expressed as the means \pm $\mathrm{SE}$. Differences between two groups were assessed using the two-tailed Student's $t$ test.

\section{Results}

PI3K/AKT signaling pathway was activated and PTEN was downregulated in mouse hemangioma endothelial cells. We examined whether AKT activation is present in EC from malignant vascular tumors. We utilized well-established malignant endothelial tumor cell lines: EOMA cells derived from a spontaneous mouse hemangioendothelioma. Increased phosphorylation of AKT Ser473,Thr308 and S6 was observed in EOMA cells compared with normal mouse lung and heart endothelial cells (obtained based on CD31-magnetic bead isolation by Carole Perrizz). The phosphorylation of AKT Thr308 is activated by PDK1(3-phosphoinositide dependent kinase-1) which is growth factor and PI3K dependent. Phosphorylation of AKT Thr308 was increased in EOMA cells. AKT Ser473 was activated mainly by rictor-mTOR which also showed high phosphorylation level in EOMA cells than control groups. S6(Ribosomal protein S6), a downstream protein of $\mathrm{AKT} / \mathrm{mTOR}$, is phosphorylated by $\mathrm{p} 70$ ribosomal protein $\mathrm{S} 6$ kinases (S6K) to regulate cell growth.It showed overactivation in EOMA cells. PTEN ( Phosphatase and tensin homolog, PTEN), as a tumor suppressor by negatively regulating $\mathrm{AKT} / \mathrm{PKB}$ signaling pathway, mutated in a large number of cancers at high frequency. In our study lower levels of PTEN were also seen in EOMA cell (Figures 1). These findings indicated that common aberrations exsit in PTEN/AKT signaling pathway and which can be targeted for the treatment of vascular tumor.

\section{Mouse shrictor clones}

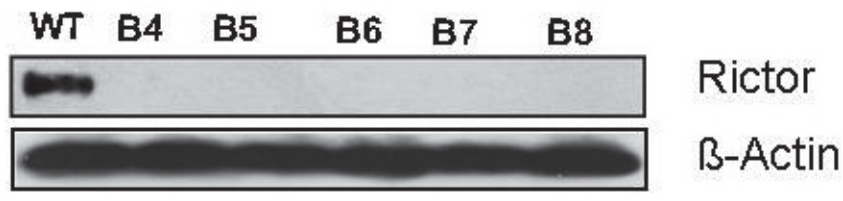

Figure 2. Rictor silencing in EOMA cells by lentiviral short-hairpin RNA. Five separate shRNA clones, shrictor \#B4,\#B5,\#B6,\#B7and \#B8 were tested. shrictor\#B8 was selected.

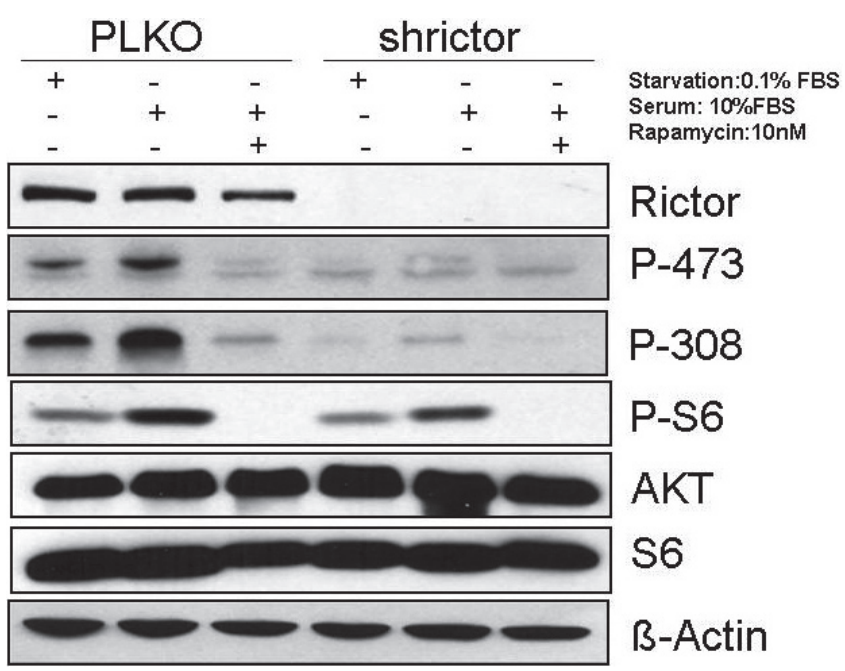

Figure 3. Western blot analysis of knocking down rictor and rapamycin treatment

in EOMA cells for AKT signaling Pathway. Decreased phosphorylated AKT occured

with $B$-actin as protein loading control.

Phosphorylation of AKT was downregulated by silencing rictor. EOMA cells were transduced with lentiviral short-hairpin RNA (shRNA) to knockdown rictor. Five targeting rictor shRNAs were used, which all expressed efficient silencing effects. Significant rictor knock-down was verified by western blot analysis (Figure 2). We selected shrictor \#B8 and set up stable rictor silencing EOMA cells for our study. PLKO and scilencing rictor EOMA cells were seperated three groups respectively. EOMA Cells of starvation group were treated by DMEM medium with only $0.1 \%$ FBS. Serum group cells was treated by normal DMEM with $10 \%$ FBS. Rapamycin(RAPA) is a inhibitor of AKT/mTOR signaling pathway. We used RAPA as a positive control. Rapamycin group cells was treated by 10nM RAPA. Western blotting results showed that loss of rictor greatly decreased phosphorylation of AKT Ser473, Thr308 and S6 compared with control PLKO cells, which was even to a similar extend like rapamycin treatment(Figure 3). As we know, AKT/mTOR/S6 signaling pathway acts the centrol role of enhancing cell growth and proliferation through various 


\section{EOMA Proliferation Assay}

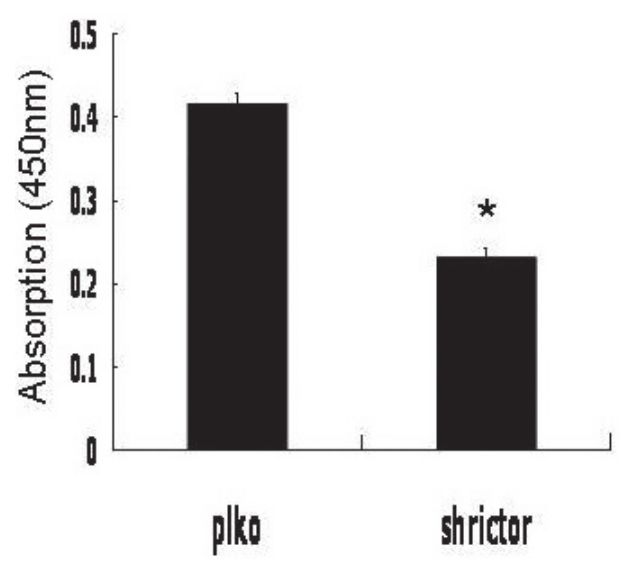

Figure 4. EOMA cells with rictor silencing affected cell proliferation. Shrictor inhibited EOMA cell proliferation compared with PLKO control $(\mathbf{p}<0.01)$.

\section{EOMA Invasion Assay}

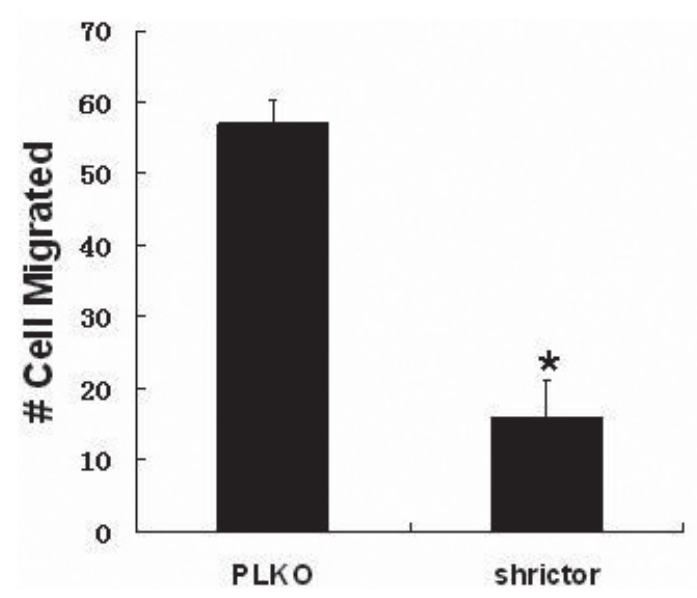

Figure 5. EOMA cells with rictor silencing affected cell invasion. Shrictor inhibited EOMA cell invasion compared with PLKO control $(\mathrm{p}<0.01)$. The invasion index was 0.28 .

mechanisms including synthesis of proteins and lipids as well as reduction of autophagy. Part of the functions are mediated by the phosphorylation and activation of AKT and S6 (a well characterized downstream effectors of AKT). We found that downregulation of rictor inhibited phosphorylation of AKT and $\mathrm{S} 6$ and thus suppressed the vascular tumor cell growth. Therefore targeting rictor becomes a good strategy for inhibiting vascular tumor.

Targeting rictor inhibited hemangioma cell proliferation in vitro. To further characterize the role of MTORC2 in vascular tumor progression, we analyzed the effect of rictor

\section{Vascular tumor growth}

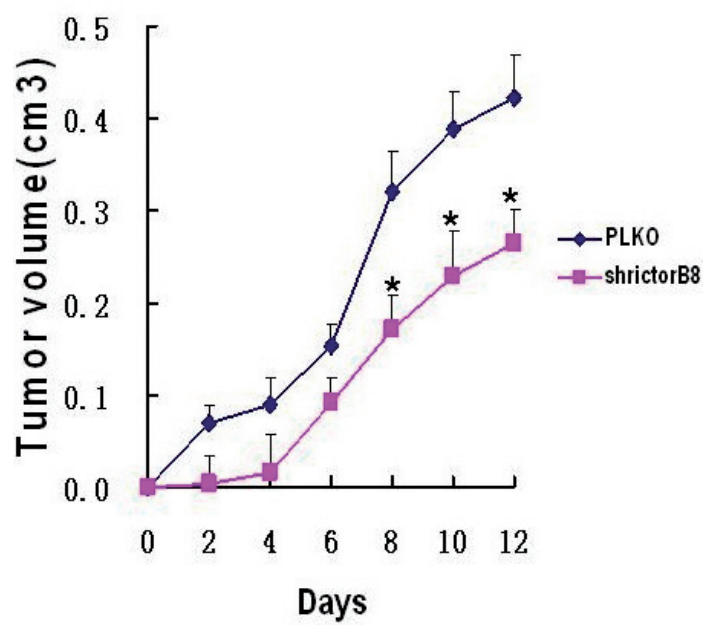

Figure 6. EOMA cells with rictor silencing injected subcutaneously affected tumor growth in nu/nu mice. Tumor size was monitored for 12 days. Rictor silencing significantly reduced tumor growth as compared to pLKO control from day8 to day12 $(\mathrm{p}<0.05)$.

knockdown on EOMA cell by WST-1 proliferation assay. We found that the proliferation of EOMA cells deficient for rictor was significantly reduced compared to control cells $(\mathrm{p}<0.01)$ (Figure 4). This result showed that rictor also greatly contributed to EOMA cell proliferation.

Targeting rictor inhibited hemangioma cell invasion in vitro. Seen from the stained cells on the membrane, the invasive cells in the EOMA with rictor knocking down was obviously lower than that of control cells $(\mathrm{p}<0.01)$. The invasion index was 0.28 (Figures 5). This finding showed that silencing rictor downregulated EOMA cell invasion capability. It indicated that rictor is greatly essential for the EOMA cells to maintain vascular tumor malignant biological behavior.

Rictor was required to form vascular tumors as xenografts in vivo. We next tested the effect of rictor knockdown on vascular tumor growth in vivo. we utilized a xenografts mouse model that knocking down rictor EOMA cells and PLKO EOMA cells were injected subcutaneously in the flank of mice respectively and tumor growth was monitored. We measured the tumor size from day 0 to day 13. Compared with control mice, vascular tumor volume in the mice with rictor knocking down was much smaller.Rictor knocking down blocked vascular tumor growth $(\mathrm{p}<0.05)$ (Figures 6). This finding showed that rictor acts a very essential role for the vascular tumor formation in vivo. Therefore, targeting rictor in vascular tumor might be a successful strategy.

\section{Disccusion}

Vascular tumors are abnormal angiogenic proliferations of neoplastic endothelial cells (EC) with a wide spectrum of 
clinical diseases ranging from common hemangioma in children to malignant angiosarcoma in adults. Although growth factors and hormonal and mechanical influences have been postulated to affect the abnormal proliferation of endothelial cells in vascular tumor, the primary, causative defect in hemangiogenesis remains unknown and no genetic alteration has been implicated.

Increased AKT activation were found in benign and malignant human vascular tumors. mTORC2 is necessary to the full phosphorylation of AKT[17]. Little is known about the functions of mTORC2. As a crucial component of mTORC2, rictor exactly acts important role in cancer progression $[18,19]$. However no study so far analyzed the specific role of rictor were found in mouse hemangioma. We found here that downregulation of rictor in mouse hemangioma cells significantly reduced cell proliferation, invasion and vascular tumor growth by the mechanism of downregulation of AKT activation partly. These results suggested that rictor acts important roles in the vascular tumor malignant biological behavior. Specifically targeting rictor may provide a novel treatment strategy for hemangioma and thus give a rationale for the development of drugs that specifically target mTORC2. Prospective studies are to be continued in order to determine whether rictor acts the same role to human vascular tumor and reveal the exact mechanism of signaling pathway by which rictor regulates cancer progression.

Acknowledgments: We would like to thank Laura E Benjamin and Qi Xue for great help with advice and experimental materials. We also thank all the members of the Laura E Benjamin labs in Harvard Medical School for assisting in protocol and cell acquisition.

\section{References}

[1] GARZON MC, HUANG JT, ENJOLRAS O, FRIEDEN IJ. Vascular malformations.Part II: associated syndromes. J Am Acad Dermatol 2007; 56: 541-564.

[2] METRY DW, DOWD CF, BARKOVICH AJ, FRIEDEN IJ. The many faces of PHACE syndrome. J Pediatr 2001; 139: 117-123.

[3] NATHAN N, THALLER SR. Sturge-Weber syndrome and associated congenital vascular disorders: a review. J Craniofac Surg 2006; 17: 724-728. http://dx.doi.org/10.1097/00001665200607000-00024

[4] ACKAH E, YU J, ZOELLNER S, IWAKIRI Y, SKURK C et al. AKT1/protein kinase Balpha is critical for ischemic and VEGF-mediated angiogenesis. J Clin Invest 2005; 115: 2119-2127. http://dx.doi.org/10.1172/JCI24726

[5] SHIOJIMA I, WALSH K. Role of AKT signaling in vascular homeostasis and angiogenesis. Circ Res 2002; 90: 1243-1250. http://dx.doi.org/10.1161/01.RES.0000022200.71892.9F

[6] BOYE E, YU Y, PARANYA G, MULLIKEN JB, OLSEN BR et al. Clonality and altered behavior of endothelial cells from hemangiomas. J Clin Invest 2001; 107: 745-752. http://dx.doi. org/10.1172/JCI11432
[7] YU Y, VARUGHESE J, BROWN LF, MULLIKEN JB, BISCHOFF J. Increased Tie2 expression, enhanced response to angiopoietin-1, and dysregulated angiopoietin-2 expression in hemangioma-derived endothelial cells. Am J Pathol 2001; 159: 2271-2280. http://dx.doi.org/10.1016/S0002-9440(10)63077$\underline{5}$

[8] BADER AG, KANG S, VOGT PK. Cancer-specific mutations in PIK3CA are oncogenic in vivo. Proc Natl Acad Sci U S A 2006; 103: 1475-1479. http://dx.doi.org/10.1073/ pnas.0510857103

[9] ITAKURA E, YAMAMOTO H, ODA Y, TSUNEYOSHI $M$. Detection and characterization of vascular endothelial growth factors and their receptors in a series of angiosarcomas. J Surg Oncol 2008; 97: 74-81. http://dx.doi. org/10.1002/jso.20766

[10] JIANG BH, ZHENG JZ, AOKI M, VOGT PK. Phosphatidylinositol 3-kinase signaling mediates angiogenesis and expression of vascular endothelial growth factor in endothelial cells. Proc Natl Acad Sci U S A 2000; 97: 1749-1753. http:// dx.doi.org/10.1073/pnas.040560897

[11] STACHER E, GRUBER-MOSENBACHER U, HALBWEDL I, DEI TOS AP, CAVAZZA A et al. The VEGF-system in primary pulmonary angiosarcomas and haemangioendotheliomas: new potential therapeutic targets? Lung Cancer 2009; 65: 49-55. http://dx.doi.org/10.1016/j.lungcan.2008.10.031

[12] SAMANIEGO F, MARKHAM PD, GENDELMAN R, WATANABE Y, KAO V et al. Vascular endothelial growth factor and basic fibroblast growth factor present in Kaposi's sarcoma (KS) are induced by inflammatory cytokines and synergize to promote vascular permeability and KS lesion development. Am J Pathol1998; 152: 1433-1443.

[13] SODHI A, MONTANER S, PATEL V, GOMEZ-ROMAN JJ, $\mathrm{LI} Y$ et al. AKT plays a central role in sarcomagenesis induced by Kaposi's sarcoma herpesvirus-encoded G protein-coupled receptor. Proc Natl Acad Sci U S A 2004; 101: 4821-4826. http://dx.doi.org/10.1073/pnas.0400835101

[14] WEBER JD, GUTMANN DH. Deconvoluting mTOR biology. Cell Cycle 2012; 15; 11(2): 236-48.

[15] POPULO H, LOPES JM, SOARES P. The mTOR Signalling Pathway in Human Cancer. Int J Mol Sci 2012; 13: 1886-918. http://dx.doi.org/10.3390/ijms13021886

[16] OH WJ, JACINTO E. mTOR complex 2 signaling and functions.Cell Cycle 2011; 10(14): 2305-16. http://dx.doi. org/10.4161/cc.10.14.16586

[17] SARBASSOV DD, GUERTIN DA, ALI SM, SABATINI DM. Phosphorylation and regulation of AKT/PKB by the rictormTOR complex. Science 2005; 307: 1098-101.

[18] ZHANG F, ZHANG X, LI M, CHEN P, ZHANG B et al. mTOR complex component Rictor interacts with PKCzeta and regulates cancer cell metastasis. Cancer Res 2010; 70: 9360-70. http://dx.doi.org/10.1158/0008-5472.CAN-10-0207

[19] MCDONALD PC, OLOUMI A, MILLS J, DOBREVA I, MAIDAN $\mathrm{M}$ et al. Rictor and integrin-linked kinase interact and regulate AKT phosphorylation and cancer cell survival. Cancer Res 2008; 68: 1618-24. http://dx.doi.org/10.1158/ 0008-5472.CAN-07-5869 